\title{
Probabilistic kingdom: problem of objectivity in contemporary science*
}

\author{
Paweł PRUSKI**
}

\begin{abstract}
In modern science the theory of probability is one of the basic tools. Scientists using probability often refer to its objective interpretation. They emphasize that their probabilistic hypotheses concern objective facts, not degrees of belief. Accordingly, the following questions arise: What is the meaning of this type of probabilistic hypothesis? Is the assumption of objectivity necessary? The paper addresses these questions by analyzing objective probability in the context of the scientific debate on determinism. Two types of arguments will be presented. On the one hand, there is the assertion that objective probability can exist only in an indeterministic world. Then, on the other hand - I analyze the assertions of those who believe in the coexistence of objective probability and determinism. As a result I show that the acceptance of deterministic and indeterministic fields as possible areas where objective probability can occur is extremely problematic. Depending on the chosen area we encounter different types of problems. Therefore, I show that a significant number of these problems are associated with the acceptance of incorrect metaphysical assumptions. And finally, I postulate that the objectivity of probability (and assumptions pertaining to it) can be reduced (without any losses) to the epistemic variant.
\end{abstract}

\section{KEYWORDS}

philosophical interpretations of probability; the objectivity of probability; probabilism; scientific realism; determinism

* This paper is the result of the research project No. 2013/09/N/HS1/00902 funded by the National Science Centre.

** Ph.D. student, Department of Philosophy, Jagiellonian University in Krakow, Poland. E-mail: minoo.music@gmail.com. 


\section{INTRODUCTION}

Currently, it is difficult to imagine a branch of science which does not use the theory of probability. In some scientific disciplines, this tool performs a fundamental explanatory role. Just look at modern economics, physics, biology, computer science, or social studies. We calculate the probability of financial gain, the occurrence of genetic mutation, the appearance of the alpha particle. This raises the elementary question - what are scientists actually saying when they use probabilities in their hypotheses? Do these assertions show what is their degree of belief in a particular thesis? Or is this an objective description of some structures of the world? What does objectivity mean in this context?

In this paper, I will answer these questions using a few philosophical ideas appearing in different discussions about probability. The first part (I) concerns the distinction between an epistemic and objective pre-interpretation of probability. In the second part (II) I analyze the distinction between so-called probabilistic assertions and probabilistic explanation. The third part of the paper (III) examines the coexistence of objective probability on a deterministic field. The final (IV) part of the paper contains some conclusions.

\section{I}

In mathematics probability is defined as a function in a defined measurement space. It is a numerical description of how likely an event is to occur or how likely it is that a proposition is true. Probability is a number between 0 and 1 , where 0 indicates impossibility and 1 indicates certainty. However, when it comes to its practical application (for example in a given field of science), there are many probability interpretations, whose adherents possess different views on the fundamental nature of probability. The main difference between them is based on assigning a different meaning to "probability". For example, in light of the so-called logical interpretation (see Carnap, 1950) we can speak of a semantic relationship between certain types of events. Conversely, the frequency interpretation (cf. von Mises, 1957) indicates that the probability of a type $A$ event can be defined as the limit of the relative frequency of the occurrences of such events in the infinite reference to type B events. An extensive discussion of the different philosophical interpretations of probability can be found in works by Thimothy Childers (Childers, 2013), Anthony Eagle (Eagle, 2010), Donald A. Gillies (Gillies, 2000) among others.

The most important consideration of this paper is the more general distinction, the so-called pre-theoretical interpretation of probability (see Gillies, 2000). This includes the distinction between epistemic and objective probabilities. In this context, the different interpretations of probability are classified into 
the above categories. It is important to correctly understand what exactly the term "pre-theoretical interpretation" means. This distinction was proposed by Gillies. Underlining this fact is very important, because in literature about the philosophical interpretation of probability, objectivity is often connected with the frequency interpretation of probability. In Gillies' pre-theoretic distinction we have a wider range (going beyond the frequency interpretation) and objectivity is characterized by reference to different types of structures. Instead of one general definition, the meaning of this pre-interpretation is explained on the basis of different characteristics of the term "objectivity". Under this proposal, we can be more specific and thus talk about different types of structures to which it refers:

(i) real observable features of the world;

(ii) real unobservable features of the world;

(iii) logical relationship between sentences.

The difference between (i) and (ii) is based on different approaches towards the structural characteristics of the world. In the first case (i) we can speak of the frequency of results for a given experiment for example (based on empirical observation). In the second case (ii) we relate to a specific type of unobservable characteristics that are attributed to the structure of the world. One example may be the tendency of a given structure to realize a certain state of things.

In the third case (iii) objectivity takes on a different form, for example we can speak of the logical relations between sentences. We postulate that they are independent of the degree of belief. The individual's beliefs are not a factor affecting the measure of probability. Logical objects (their relations) have an independent status. This type of objective characteristic can be found in John Maynard Keynes (Keynes, 1921) and Rudolf Carnap's early works (Carnap, 1950). Especially in Logical foundations of probability (Carnap, 1950), we can read: "That the objective logical concept meant by Keynes is the same as we call logical probability , i.e., the logical concept of confirmation" (Carnap, 1950: 44).

In this context, logical probability is based on the logical relationships between elements of a semantic system. This becomes more clear when we look at the general idea of Carnap's goal - to create the logic of induction in which the main tool is logical probability. According to Carnap, deductive relations are analytic (are independent of reality) in the sense that whatever is contained in the conclusion of a valid deductive argument is also contained in the premise. Carnap wanted a similar notion of containment for his probabilistic system. Of course in many definitions the logical interpretation identifies probability with the degree of rational belief (it is assumed that given the same evidence, all rational human beings will entertain the same degree of belief in a hypothesis or prediction). In his later work (Carnap, 1971), Carnap advocates this view, and his system is very close to decision theory. But in Logical foundations of probability (Carnap, 1950), "rational belief" is represented by a pure and simple 
logical function and the result depends only on logical and semantic components, e.g., relationships between predicates or more precisely - the so-called measure function. In this light, we can (according to the author) define this proposal as objective. We must remember that in this interpretation we are using meta-language and as Carnap himself often emphasizes - our hypotheses say nothing about the world, but only about certain relations within the semantic system.

On the other hand, Gillies distinguishes epistemic probability. This is defined as a measure of our uncertainty regarding the possibility of the occurrence (or lack thereof) of a given event. It is a characteristic of our beliefs about the chance of uncertain events occurring.

Epistemological interpretations of probability take probability to be concerned with the knowledge or belief of human beings. On this approach probability measures degree of knowledge, degree of rational belief, degree of belief, or something of this sort (Gillies, 2000: 2).

The epistemic approach is usually connected to the so-called subjective interpretation of probability (see Ramsey, 1926; de Finetti, 1931). Probability is characterized as the degree of belief of a rational individual. The degree of belief has been interpreted as "the price at which you would buy or sell a bet that pays 1 unit of utility if E, 0 if not E". According to this interpretation, the key fact is that the function of beliefs is consistent with the axioms of probability. A specific set of conditions is imposed onto the function of beliefs. As a result, epistemic probability is a particularly important tool used in different branches of science. But for certain reasons, many scientists postulate that within their fields it is necessary to use objective probability.

Let's examine the position of supporters of objective probability and analyze three sentences that use references to probabilities. Thanks to this, we will be able to see that the term "probability" does not have the same meaning in all cases. Here are three examples:

(a) There is a $70 \%$ chance that the leader of North Korea Kim Jong Un will increase the military budget.

(b) The probability that dinosaurs became extinct 65 million years ago as a result of an asteroid hitting the Earth is $70 \%$.

(c) The probability that an atom of radium will disintegrate in t 1 time is $70 \%$. The above examples are characterized by varying degrees of accuracy and relate to different fields of science. Their common element is a reference to 
probability. Can all these assertions be treated as equal? According to many philosophers - no. Jonathan Schaffer (Schaffer, 2007) authored one of such classifications, which requires a distinction between these assertions. According to the author we should distinguish between the so-called explanation through probability and a probabilistic explanation.

All "less strong" applications of probability are considered as an explanation through probability. These are applications in which, thanks to additional facts, the probability could be reduced to a non-probabilistic characteristic. According to Schaffer, these types of statements employ a weak use of the term "probability". From the examples above we can group (a) and (b). In the first case (a) additional information on the conduct of the North Korean leader would be required. For example, we may ask the Korean leader and we could get the answer "Yes, I will increase the military budget". In the second case (b) if we went back in time to 65 million years ago we would know the cause of dinosaur extinction. For example, after seeing what actually happened, we could say: "No, dinosaurs did not become extinct due to an asteroid impact". As a result (a) and (b) could be presented in a non-probabilistic form.

The second type of assertions are probabilistic explanations. This category applies to all assertions irreducible to a non-probabilistic form. According to Schaffer, this criterion is extremely rigorous. Most statements (including those occurring in the world of science) in the light of the characteristic would be rejected. Only statements about the processes occurring on the indeterministic field are permitted (e.g., quantum processes). According to Schaffer, all other probabilistic assertions (without such a strong reference) should fall into the first group. And so, the example assertion (c) can be defined as a probabilistic explanation. Irreducibility stems from the role probability plays in quantum mechanics.

Should we agree with the above differentiation? On the one hand, without a doubt we should separate the common and scientific understandings of probability. A similar distinction was proposed by Carnap (Carnap, 1950). This is a division between the so-called explicandum and explicatum. The first group consists of colloquial terms, as well as those which are in a pre-scientific state. The second group (explicatum), according to Carnap, includes more precise terms as are applied in science. Importantly, the two groups are not mutually exclusive - it is important to differentiate the area in which we use the term (in this case probability).

In this case should we agree to the second part of Schaffer's proposal? Can objective probability be realized only on the indeterministic field? Many philosophers do not agree. Before proceeding further to the part devoted to the coexistence of objective probability and determinism - it's worth considering for a moment the indeterministic field. Is it actually a non-controversial field for this type of probability? 
Schaffer refers to quantum processes, but this does not help him avoid the complexity of interpretation. It is worth mentioning the problems of measure and reference. In their light a problem arises due to the discrepancy between the assumed model and empirical experience (function characteristic). Consequently, we do not have an explicit reference between the probabilistic assertions and an ontological structure. To summarize this section of the paper, we have seen various meanings that can be associated with objective probability. We now also see in which cases we can talk about the strongest use of the term. The outlined examples lead us to the division into deterministic (examples $\mathrm{a}$ and $\mathrm{b}$ ) and indeterministic (example c) field of scenes. Let us check what the relationship is between these areas and objective probability.

\section{III}

Determinism is, roughly speaking, the idea that every event is necessitated by antecedent events and conditions together with the laws of nature. It is deeply connected with our understanding of the physical sciences and their explanatory ambitions. To be more precise we should look at the definition:

Determinism - doctrine that every event has a cause. The usual explanation of this is that for every event, there is some antecedent state, related in such a way that it would break a law of nature for this antecedent state to exist yet the event not to happen (Blackburn, 1994: 103).

In light of the above definition a coexistence of determinism and probability seems to be quite a controversial thesis. How is it possible that probability occurs in a world where everything is connected by a necessary chain of causality (cause and effect)? This question was raised by many philosophers:

Today I can see why so many determinists, $[. .$.$] believe in a subjectivist interpretation$ of probability: it is, in a way, the only reasonable possibility which they can accept; for objective physical probabilities are incompatible with determinism [...] (Popper, 1982: 105).

Nevertheless, the possibility of the coexistence of determinism and objective probability has its supporters, the so-called compatibilists. The main representatives of this view include Barry Loewer (Loewer, 2001) and Carl Hoefer (Hoefer, 2007). These philosophers refer mainly in their arguments to examples from classical statistical mechanics. In their opinion, classical statistical mechanics are interpreted as deterministic, but in this scientific field the main tool is the theory of probability. For example, we can calculate (using probability) the spontaneous reversion molecules of a gas in a bottle. In this context, 
the compatibilist assumes that we are dealing with objective probability and not a degree of belief.

In spite of this dissonance, compatibilists seek to maintain the objectivity of probability. The source of this requirement is derived from the characteristics of empirical sciences. If science is to be objective, it should not be based on the subjective beliefs of the individual. According to compatibilists, subjectivation is incompatible with the basic principles of scientific methodology. In consequence, probability in various fields of science is an explaining factor for compatibilists this is a premise which proves the objectivity of applied probability.

An interesting summary of the evidence referred to by the compatibilists is provided by Schaffer. It features three arguments in defense of compatibilists.

The paradigm case argument - in a deterministic world, there are some specific cases relating to randomness. We attribute fixed, objective probability to them.

(P1) There are coin flips in deterministic worlds;

(P2) Coin flips involve a 0.5 objective chance that a heads landing will occur, at the world in question, at the time of flipping:

(C) There are objective chance values other than 0 or 1 in deterministic worlds.

The incompatibilist should deny (P2), and should distinguish between objective chance, and merely epistemic chance. Of course, this is not to deny that objective information (such as frequency data, and physical information concerning the asymmetries in the coin) can impact the chance of landing heads.

The nonreductionist argument - in a deterministic world there are macro events. The macro space is autonomous (in relation to the micro) and has associated rights, and thus an objective probability, which is not necessarily reducible to micro events (occurring on an indeterministic field).

(P1) There are macro-events in deterministic worlds;

(P2) The macro-realm has an independent reality with independent laws and chances;

(C) There may be macro-chances in deterministic worlds.

The incompatabilist should still deny (P2), however precisely explicated. That is, even granting some form of "independent reality" to the macro-realm, the incompatibilist should still deny the independence of macro-chances.

The CSM argument - referring to classical statistical mechanics classical statistical mechanics do not exclude that in a deterministic world there are random events. Objective probability plays an explanatory role there (e.g., in thermodynamics).

(P1) CSM postulates nondegenerate chances in a deterministic world;

(P2) Such chances play a role in explanations;

(C) Such chances must be objective chances. 
The incompatibilist should deny (C). There should be a distinction between the probabilistic explanation, in which objective chances play an explanatory role, and the probability of explanation, which is merely an ignorance measure over various non-chance explanatory paths.

At their foundation, these arguments are based on a different understanding of objective probability. Therefore, according to Schaffer, four types of objective probabilities should be distinguished:

d1) Deterministic micro-posterior chance.

d2) Deterministic macro-posterior chance.

d3) Deterministic micro-initial chance.

d4) Deterministic macro-initial chance.

This division is based on two main criteria. First one concerns the distinction on the basis of the field of physical processes: micro (e.g., the disintegration of a radium atom in $\mathrm{x}$ time) - types (d1) and (d3) and macro (e.g., a coin toss) - types (d2) and (d4).

The second is based on a temporal classification. The featured variants are: initial - types (d3) and (d4), posterior - types (d1) and (d2). The first relates to the initial moment of the universe (a reference to entropy). The second refers to a specific time before the occurrence of a given event.

A summary of the dependencies between three compatibilist arguments and a certain variation of deterministic chance (d1-d4) is shown in the following table.

Tab. 1. Summary of arguments in favour of compatibilism with different types of chance

\begin{tabular}{|c|c|c|c|c|}
\cline { 2 - 5 } \multicolumn{1}{c|}{} & $\begin{array}{c}\text { Deterministic } \\
\text { micro-posteriori } \\
\text { chance }\end{array}$ & $\begin{array}{c}\text { Deterministic } \\
\text { macro-posteriori } \\
\text { chance }\end{array}$ & $\begin{array}{c}\text { Deterministic } \\
\text { micro-initial } \\
\text { chance }\end{array}$ & $\begin{array}{c}\text { Deterministic } \\
\text { macro-initial } \\
\text { chance }\end{array}$ \\
\hline $\begin{array}{c}\text { The paradigm } \\
\text { case argument }\end{array}$ & - & + & - & - \\
\hline $\begin{array}{c}\text { The non reduc- } \\
\text { tionist argument }\end{array}$ & - & + & - & + \\
\hline $\begin{array}{c}\text { The CSM } \\
\text { argument }\end{array}$ & - & - & - & + \\
\hline
\end{tabular}

So we can see that selected types of arguments refer to the specific interpretations of objective probability. Of course, opponents of this view, incompatibilists, deny the above thesis. The probability occurring in these types of assertions should be characterized as a subjective degree of an individual's belief. In other words - epistemic probability. Counterarguments are primarily focused 
on demonstrating the contradictions of the above arguments. However, it is quite interesting to take a slightly different route and to see why we should even postulate the existence of objective probability.

As we have seen, the acceptance of objective probability is associated with a whole group of problems. It is not difficult to notice that a large part of them could be resolved by agreeing to an epistemic version of probability. So why do so many scientists say that objective probability is necessary? In response compatibilists talk of three types of premises defending an objective probability (thereby negating the epistemic): usability in science, informative objectivity, reference to reality.

Usability in science. In this premise, scientific theories should be associated with an objective factor. Rejection of objectivity means narrowing the scope of theory as well as - in the ultimate case - its usefulness. But is this really a good argument for proponents of objective probability? I think not. It is not difficult to see that the use of a reference to the epistemic interpretation does not mean a limitation on its usefulness. This is evidenced by numerous examples appearing within decision theory, demography and economics, among others. In a more detailed reference it is enough to analyze the tools used within the so-called subjective Bayesianism (used in certain areas of quantum mechanics). Even in branches of science that are closer to social science, probability can be interpreted in both ways. For example in utility theory (part of decision theory) probability is often interpreted as measuring individual degrees of belief. But nothing in the formalism of utility theory forces this interpretation on us. We could instead interpret probabilities as objective chances or as the degrees of belief that are warranted by the evidence, if we thought these were a better guide to rational action. The difference is mainly based on the selection of a suitable formal system and both variants are equally useful. In some cases it is better to use one type of formalism, in others it will be more appropriate to use another.

Informative objectivity. The information expressed in a probabilistic hypothesis has the same value, independent of some subjective beliefs. But, an epistemic understanding of probability may be associated with objective information. Much like in the previous case, we can refer to the Bayesian principle of conditioning. To further justify this thesis, we can go back to Carnap's example. As we saw in the first part of this paper he began his work on pure logical, objective interpretation and later reformulated the theory to subjective interpretation. But once again we must remember a few assumptions. The new version of this theory was still based on a semantic-logical concept without losing any of its informative precision. In practice, this means an additional 
parameters, such as formal conditions of rationality and decision making. Both the first (objective) and second (subjective) versions have their pros and cons, which are not associated with the problem of a lack of objectivity of information. Of course in both theories the results will be different, but this difference is based only on logical and semantic assumptions. Therefore, I think that using the argument of informative objectivity to defend the objectivity of probability is a mistake. We receive the same informational objectivity for both objective and epistemic probabilities.

Reference to reality. At this point, it is good to return to three different types of structures (i) - (iii) to which the term "objective" can be referred to. As we can see an ontological reference is equally problematic in both types of probability classification. Objective understanding of probability does not have any significant advantage here. On an indeterministic field (in quantum mechanics) there are a whole range of problems associated with reference. In Chapter III we saw how many problems arise when we try to combine objective probability with different types of theories (deterministic and indeterministic). In reference to the structures mentioned above, we still have the third option (iii). Does the connection between objectivity and the "logical relationship between sentences" solve our problem? In my opinion, here we can actually talk about objectivity, but if we look at it a little closer - we do not obtain any benefits. We are able to agree that e.g., logical and semantic relations are objective, but we must remember that we are not really talking about the empirical world. Our description applies only to the system and its structure. The degree to which this system describes some part of reality depends on the theory. This is a different, difficult philosophical problem concerning the relationship between theory and the described structure. In this area we can, e.g., question whether nature can be described by mathematics, etc. But, concluding this part, I think in this light the objective interpretation of probability also does not provide any benefits. It has the same status as an epistemic interpretation.

If we accept the above assumptions, we can ask an additional question - is it necessary to use objective probability? Or should we adopt a radical position and reject the objectivity of probability? An interesting intuition about this fact comes from de Finetti:

The abandonment of superstitious beliefs about the existence of the Phlogiston, the Cosmic Ether, Absolute Space and Time, [...] or Fairies and Witches was an essential step along the road to scientific thinking. Probability, too, if regarded as something endowed with some kind of objective existence, is no less a misleading misconception, an illusory attempt to exteriorize or materialize our true probabilistic beliefs (de Finetti, 1974: X).

I think that it is not necessary to adopt such a radical position. In my opinion, the basis of the above problems is a few incorrect assumptions. First of all, 
the pre-theoretical division proposed by Gillies generates unnecessary metaphysics. An attempt to categorize individual interpretations of probability as objective or epistemic is philosophically interesting, but has no scientific value. Of course, the authors of some interpretations use this term (e.g., Carnap), but in most cases they do not create ontological references. Rather, their goal is to show independence from a certain group of unwanted factors (e.g., the psychological) that could deform theory results. Postulating an independent, objective probability in some sphere of ontology is simply misleading. Secondly, creating ontological references transfers the discussion to another field, e.g., regarding the relationship between theory and its representation. This problem is particularly pronounced in the third part of the work (III). Probability is located within specific ontological structures - deterministic or indeterministic. As in the previous case, we are again moving from the scientific to the metaphysical level. In addition to the above assumptions (usability, informativity, references), as we have seen, the adoption of objective probability does not bring any benefits. The same postulates can be realized by epistemic versions. To sum up, I think that combining the objectivity of probability with an ontological structure is a mistake.

\section{BIBLIOGRAPHY}

Blackburn, S. (1994). The Oxford dictionary of philosophy. Oxford: Oxford University Press. Carnap, R. (1950). Logical foundations of probability. Chicago: University of Chicago Press. Carnap, R. (1971). A basic system of inductive logic. Part I. Oakland: University of California Press.

Childers, T. (2013). Philosophy and probability. Oxford: Oxford University Press.

Eagle, A. (2010). Philosophy of probability: Contemporary readings. London: Routledge.

de Finetti, B. (1974). Theory of probability. Hoboken: John Wiley \& Sons.

de Finetti, B. (2008). Philosophical lectures on probability. Berlin: Springer Verlag.

Gillies, D.A. (2000). Philosophical theories of probability. London: Routledge.

Hoefer, C. (2007). The third way on objective probability: A sceptic's guide to objective chance. Mind, 116(463), 549-596.

Keynes, J.M. (1921). A treatise on probability. London: Macmillan and co.

Loewer, B (2001). Determinism and chance. Studies in History and Philosophy of Modern Physics, 32, 609-620.

Popper, K. (1982). Quantum theory and the schism in physics. New Jersey: Rowman and Littlefield.

Ramsey, F.P. (1926). Truth and probability (pp. 156-198). In: R.B. Braithwaite (Ed.). Foundations of mathematics and other essays. London: Kegan, Paul, Trench, Trubner, \& Co..

von Mises, R. (1957). Probability, statistics and truth. (2 ${ }^{\text {nd }}$ Eng. ed.). Dover: George Allen \& Unwin.

Nau, R.F. (2002). De Finetti was right: probability does not exist. Theory and Decision, 51, 89-124.

Schaffer, J. (2007). Deterministic chance?. The British Journal for the Philosophy of Science, 58(2), 113-140. 
